\title{
Changes in Oxidative Stress and Inflammatory Biomarkers in Fragile Adults over Fifty Years of Age and in Elderly People Exclusively Fed Enteral Nutrition
}

\author{
Maria D. Mesa, ${ }^{1}$ Josune Olza, ${ }^{1}$ Carolina Gonzalez-Anton, ${ }^{1}$ \\ Concepcion M. Aguilera, ${ }^{1}$ Rosario Moreno-Torres, ${ }^{2}$ Africa Jimenez, ${ }^{3}$ \\ Antonio Perez de la Cruz, ${ }^{2}$ Azahara I. Ruperez, ${ }^{1}$ and Angel Gil ${ }^{1}$ \\ ${ }^{1}$ Department of Biochemistry and Molecular Biology II, Institute of Nutrition and Food Technology "Jose Mataix", \\ Biomedical Research Centre, Health Science Technological Park, University of Granada, Avenida del Conocimiento s/n, \\ 18016 Granada, Spain \\ ${ }^{2}$ Clinical Nutrition and Dietetic Unit, University Hospital Virgen de las Nieves, Avenida de las Fuerzas Armadas 2, \\ 18014 Granada, Spain \\ ${ }^{3}$ Vegenat, S.A. Research and Development Department, Ctra Badajoz-Montijo, $k m$ 24,9, \\ Pueblonuevo del Guadiana, 06184 Badajoz, Spain \\ Correspondence should be addressed to Azahara I. Ruperez; azahararuperez@ugr.es
}

Received 7 April 2015; Revised 14 August 2015; Accepted 16 August 2015

Academic Editor: Gabriele Saretzki

Copyright (C) 2016 Maria D. Mesa et al. This is an open access article distributed under the Creative Commons Attribution License, which permits unrestricted use, distribution, and reproduction in any medium, provided the original work is properly cited.

\begin{abstract}
We aim to evaluate whether exclusive feeding of an enteral formula enriched with $n$-3 long chain polyunsaturated fatty acids ( $n$-3 LC-PUFA) affects oxidative stress and the antioxidant defence system and may improve the levels of some relevant inflammatory, and cardiovascular biomarkers in frail adults over fifty years of age and in elderly subjects. Fifty-five patients were divided into two groups and were exclusively fed a newly designed normoproteic and isocaloric enteral formula enriched with eicosapentaenoic $(98 \mathrm{mg} / \mathrm{d})$ and docosahexaenoic acids $(46 \mathrm{mg} / \mathrm{d})(n=26)$ or a reference enteral diet $(n=29)$. Oxidative, inflammatory and cardiovascular risk biomarkers and red blood cell fatty acid profiles were determined at the beginning and after 90 and 180 days of feeding. The $n$-3 LC-PUFA percentage tended to be higher $(P=0.053)$ in the experimental group than in the reference group. Administration of the $n$-3 LC-PUFA diet did not increase oxidative stress or modify plasma antioxidant capacity but decreased antioxidant enzymatic activities. MMP-9 plasma concentration decreased with both formulae, whereas tPAI-1 tended to decrease $(P=0.116)$ with the administration of the experimental formula. In conclusion, administration of the new $n$ - 3 LC-PUFA-enriched product for 6 months did not negatively alter the oxidative status and improved some cardiovascular risk biomarkers.
\end{abstract}

\section{Introduction}

Active ageing is becoming an important topic for the process of optimizing opportunities for health, participation, and security to improve the quality of life of aged people. Lifestyle changes, including physical activity and a healthy suitable diet, are the most important strategies available to modulate health outcomes [1,2]. Ageing is a physiological process characterized by a number of changes in the body and which impair the ability to respond to stress, which increases the risk of diseases such as cardiovascular disease (CVD) and other chronic diseases $[3,4]$. Accumulating evidence suggests that age-related diseases are associated with increased oxidative stress $[5,6]$, an impaired antioxidant defence system [7], and deregulated immune and inflammatory responses $[3,7,8]$. Moreover, silent inflammation during ageing is evidenced by increased circulating levels of proinflammatory cytokines, acute phase proteins, and organ dysfunction that leads to the development of several diseases [3]. In addition, the close association between risk of malnutrition and functional impairment requires nutritional intervention strategies to prevent the development and progression of several 
diseases [3]. Fragile adults affected by severe chronic diseases also exhibit an altered oxidative status [9] and a derangement in the antioxidant defence system [10].

Enteral nutrition (EN) containing specific nutrients and bioactive compounds are used in a number of situations when oral feeding is not an option, particularly in fragile, aged patients $[11,12]$. In general, enteral formulae contain different lipid mixtures and represent a reliable supply of energy and essential fatty acids. Lipid emulsions made from soybean oil in various combinations with medium-chain triacylglycerols (TAG), olive oil, and fish oil are widely used according to the purpose of the formula [13]. One of these emulsions includes the use of purified $n-3$ long chain polyunsaturated fatty acids ( $n$-3 LC-PUFAs), that is, eicosapentaenoic acids (EPA), and docosahexaenoic acids (DHA) [14]. These fatty acids play an important role in modulating the inflammatory response and alleviating symptoms in patients at high risk of developing inflammatory diseases [15-18]. Recently, two systematic reviews demonstrated that dietary $n-3$ fatty acids are associated with lower levels of inflammation and endothelial activation plasma biomarkers in patients with CVD and other chronic and acute diseases [19]. Moreover, dietary $n$-3 PUFAs are associated with improved systemic insulin sensitivity [20]. An appropriate balance between $n$ 3/n-6 PUFAs in the diet diminishes the risk of CVD due to the anti-inflammatory properties of $n$-3 PUFAs [21] and the proinflammatory character of $n-6$ PUFAS such as $\gamma$-linolenic acid and dihomo- $\gamma$-linolenic acid, upregulated in the modulation of the inflammatory response [22]. However, once incorporated into cell membranes, $n$-3 LC-PUFAs are more susceptible to oxidation [23], which could trigger oxidative stress-derived alterations, leading to increased inflammatory processes [4]. Therefore, more evidence is needed to establish dietary recommendations for these fatty acids in the elderly [19].

Our research group previously observed that feeding exclusively with an EPA and DHA-enriched enteral formula allowed $n$-3 LC-PUFA incorporation into total plasma lipids (specifically EPA in the phospholipids and cholesterol ester fractions) and normalized plasma TAG levels after 3 months of feeding [24]. However, the potential benefits of dietary n-3 LC-PUFA administration on oxidative stress and inflammatory and cardiovascular risk biomarkers using that enteral formula have not been previously addressed. Therefore, the present study determined the influence of that enteral formula enriched in n-3 LC-PUFA on oxidative, inflammatory, and cardiovascular risk biomarkers and the impact on the antioxidant defence system following exclusive enteral feeding for 6 months in fragile adults aged over fifty years and in elderly patients.

\section{Methods}

2.1. Subject Selection and Allocation. Fifty-five outpatients (mean age $79 \pm 1$ years and range 52 to 97 years; $n>$ $50<65=6$ and $n>65=49$ ) from the Unit of Clinical Nutrition at the University Hospital Virgen de las Nieves (Granada) were recruited and asked to participate in the study. The inclusion criteria were the prescription of total EN for at least 6 months and voluntary consent for participation. The exclusion criteria were an unstable clinical situation, fatal illness, refusal to participate in the study, or being enrolled in another clinical trial. Initially, we selected fifty-five outpatients. Concomitant diseases were mainly cognitive deficits and Alzheimer's disease, cerebrovascular diseases and cardiovascular events, and cancer in few occasions. Medications used by these patients were mainly gastric protectors, psychodrugs, anticoagulants and antihypertension medication, and, with less frequency, diuretics, analgesics, antiarrhythmics, and antidiabetic drugs in few occasions. The patients were randomly allocated into two groups: experimental $(n=26)$ and reference $(n=29)$. In the experimental group, five patients withdrew, four died, two changed their diets, and one no longer required EN. In the reference group, five patients withdrew, three died, and two changed their diets. Thus, at the end of the intervention, thirty outpatients (age $79 \pm 2$ years; range 52 to 94 years) (experimental group $(n=14)$ and reference group $(n=19)$ ) completed this study. Mean baseline tricipital skinfold was $17.6 \mathrm{~mm}$ and $17.5 \mathrm{~mm}$ for the experimental and the reference groups, respectively, while midarm circumference was $24 \mathrm{~cm}$ and $25 \mathrm{~cm}$ for the experimental and the reference groups, respectively.

The study was approved by the Ethics Committee of the University Hospital Virgen de las Nieves from Granada. All procedures were performed in accordance with the institutional guidelines following the IHC Harmonized Tripartite Guideline for Good Clinical Practice in accordance with the Helsinki Declaration of the World Medical Association: Ethical Principles for Medical Research on Human Beings (revised in Edinburgh, October 2000). All individuals or subjects' caregivers provided written informed consent prior to their inclusion in the study.

2.2. Study Design and Performance. The present study was a randomized, experimental, prospective, and intention-totreat study (6-month duration) with two parallel groups that were fed exclusively with EN. In the experimental group, a newly designed normoproteic $(40 \mathrm{~g} / 100 \mathrm{~mL})$ and isocaloric enteral formula (T-Diet Plus, Vegenat S. A., Badajoz, Spain) containing $75 \mathrm{mg} / \mathrm{L}$ EPA and $35 \mathrm{mg} / \mathrm{L}$ DHA was administered. In the reference group, a standard normoproteic $(4 \mathrm{~g} / 100 \mathrm{~mL})$ and isocaloric enteral formula (Jevity, Abbot Laboratories, USA) (without $n$-3 LC-PUFAs) was administered. Table 1 presents the nutritional composition of the experimental and reference diets.

The subjects were provided $1500 \mathrm{~mL}$ of the diet, which guaranteed their daily energetic and nutritional requirements. The overall mean daily intake was $1266 \pm 60 \mathrm{~mL} / \mathrm{d}$ (mean \pm SEM) for the T-Diet Plus group and $1362 \pm 50 \mathrm{~mL} / \mathrm{d}$ for the reference group. There was no difference in the daily intake between the groups. The mean EPA and DHA intake of subjects receiving the T-Diet Plus was approximately 94 and $44 \mathrm{mg} / \mathrm{d}$, respectively. Administration of both products was performed as a bolus using a nasogastric feeding tube with a large-bore syringe or via an ostomy. To maintain the optimal hydration state, all patients received $1000-1200 \mathrm{~mL}$ of water daily. 
TABLE 1: Nutritional composition of the experimental and reference diets.

\begin{tabular}{lcc}
\hline & Experimental & Reference \\
\hline Energy (kcal/dL) & 100 & 103 \\
Proteins (g/dL) & 4.0 & 4.0 \\
Carbohydrates (g/dL) & 12.3 & 14.05 \\
Fat (g/dL) & 3.9 & 3.47 \\
$\quad$ Saturated (g/dL) & 0.93 & 0.70 \\
$\quad$ Monounsaturated (g/dL) & 2.02 & 2.00 \\
$\quad$ Polyunsaturated (g/dL) & 0.95 & 0.77 \\
$\quad$ Essential fatty acids (g/dL) & 0.85 & 0.76 \\
$\quad n-3$ LC-PUFA (mg/dL) & 110 & - \\
Vitamin E (mg/L) & 10 & 0.23 \\
$n-6: n$-3 ratio & $6.3: 1$ & $10: 1$ \\
Fibre (g/L) & 1.70 & 1.44 \\
Total minerals (g/dL) & 0.71 & 0.57 \\
\hline
\end{tabular}

The product contains a vitamin complex to satisfy $100 \%$ of the recommended vitamin intake for the elderly assuming a daily intake of $1500 \mathrm{kcal}(6276 \mathrm{~kJ})$. $n$-3 LC-PUFA: $n$-3 long chain polyunsaturated fatty acids.

2.3. Blood Samples. Fasting blood samples were collected between 8:00 and 10:00 am at time 0 and after 90 and 180 days. The serum and plasma (EDTA-coated tubes) were separated by centrifugation $(15 \mathrm{~min}$ at $1750 \times \mathrm{g}$ ) and were immediately frozen at $-80^{\circ} \mathrm{C}$. The buffy coat was mixed with RPMI $(1: 1)$, layered onto Histopaque, and centrifuged to obtain lymphocyte isolation $\left(700 \times \mathrm{g}, 30 \mathrm{~min}\right.$ at $\left.20^{\circ} \mathrm{C}\right)$. Once isolated, the lymphocytes were washed with RPMI $10 \%$ FBS and were frozen gradually at $-80^{\circ} \mathrm{C}$ in FBS $10 \%$ DMSO until use. Finally, the red blood cells (RBCs) were washed three times with $\mathrm{NaCl} 0.9 \%$, lysed with cold water, and frozen at $-80^{\circ} \mathrm{C}$ until use.

2.3.1. Plasma Lipids. Plasma TAG, total cholesterol (TC), low-density lipoprotein cholesterol (LDLc), apolipoprotein B (apoB), high-density lipoprotein cholesterol (HDLc), and apolipoprotein A-I (apoA-I) concentrations were determined by standardised spectrophotometric techniques using a Roche Hitachi Modular DDP clinical analyser system (Roche Diagnostics España, S. L., Barcelona). All parameters were analysed at the laboratory of the Virgen de las Nieves University Hospital (Granada).

2.3.2. Oxidative Stress Biomarkers. An enzymatic immunoassay for the quantitative determination of plasma oxidized low-density lipoprotein (oxLDL) was used (Cat. number BI-20042, Biomedica Medizinprodukte GmbH \& Co KG, Vienna, Austria). After LDL isolation [25], LDL oxidation was induced by incubating with $20 \mu \mathrm{M} \mathrm{CuSO}_{4}$. LDL susceptibility to oxidation was determined as previously described [26]. Conjugated dienes were measured after $300 \mathrm{~min}$, and the amount of lipid hydroperoxides was determined after $4 \mathrm{~h}$ of incubation. Peripheral blood lymphocyte DNA damage was determined using the double-strand break analysis (termed the COMET assay) as previously described [27]. Briefly, frozen lymphocytes were lysed, and the cellular proteins were removed. DNA electrophoresis was performed to allow for separation of the two DNA strands. 6-diamidino-2phenylindole (DAPI)-stained nucleotides were examined with a UV microscope with an excitation filter of $435 \mathrm{~nm}$ and at 400x magnification.

2.3.3. Nonenzymatic Antioxidant Defence System. Plasma total antioxidant capacity (TAC) was assessed using a spectrophotometric commercial assay kit (Cat. number 709001, Cayman, MI, USA). After extraction with 1-propanol, the plasma concentrations of retinol, tocopherol, and coenzyme $\mathrm{Q}_{10}\left(\mathrm{CoQ}_{10}\right)$ (oxidized, reduced, and total) were determined by high pressure liquid chromatography coupled to an electrochemical detector (HPLC-EC) according to Battino et al. [28]. The amount of beta-carotene was also determined after extraction with 1-propanol in an HPLC system attached to a multiwavelength ultraviolet detector set at $450 \mathrm{~nm}$. All compounds were identified by predetermining the retention times of individual standards.

2.3.4. Enzymatic Antioxidant Defence System. The haemoglobin $(\mathrm{Hb})$ concentration in the blood samples was determined spectrophotometrically by the colorimetric cyanmethemoglobin method [29] using Sigma Diagnostic reagents. RBC antioxidant enzyme activities were determined spectrophotometrically as described previously with slight modifications for microplate analysis. RBC catalase activity was assayed as described by Aebi [30] and was expressed as $\mathrm{nmol} /(\mathrm{L} \cdot \mathrm{gHb})$. RBC superoxide dismutase (SOD) activity was assayed according to the methods of McCord and Fridovich [31] and was expressed as U/mg Hb. RBC glutathione reductase (GR) activity was assayed by the method of Carlberg and Mannervik [32] and was expressed as $\mathrm{U} / \mathrm{g} \mathrm{Hb}$. RBC glutathione peroxidase (GPx) activity was assayed by the coupled enzyme procedure with tert-butyl hydroperoxide as a substrate [33] and was expressed as U/g $\mathrm{Hb}$.

2.3.5. Inflammatory and Cardiovascular Risk Biomarkers. MILLIPLEX kits (Linco Research, MO, US) were used with a Luminex 200 System (Luminex Corporation, TX) to determine the amount of soluble intercellular adhesion molecule- (sICAM-) 1 (CV: 7.9\%), soluble vascular cell adhesion molecule- (sVCAM-) 1 (CV: 4.5\%), soluble endothelial selectin (sE-selectin) (CV: 11.2\%), matrix metalloproteinase(MMP-) 9 (CV: 6.8\%), myeloperoxidase (MPO) (CV: 12.3\%), total plasminogen activator inhibitor- (tPAI-) 1 (CV: 6.6\%) (Cat. HCVD1-67AK), interleukin- (IL-) 6 (CV: 7.8\%), tumour necrosis factor- (TNF-) $\alpha$ (CV: 7.8\%), and monocyte chemotactic protein (MCP)-1 (CV: 7.9\%) (Cat. HADK2-61 K-B). Endothelin-1 was analysed by ELISA (Cat. number BI-20052, Biomedica Medizinprodukte $\mathrm{GmbH}$ \& Co KG, Vienna, Austria). High sensitive $\mathrm{C}$ reactive protein (hsCRP) was quantified with a turbidimetric assay (Dade Behring Inc., Deerfield, IL).

2.3.6. Quantification of Fatty Acids in Red Blood Cells. RBC lipids were dissolved with isopropanol (25 mg/L BHT) and 
TABLE 2: Plasma lipid profiles elderly patients were fed exclusively by total enteral nutrition with the experimental and reference diets for $90 \mathrm{~d}$ and $180 \mathrm{~d}$.

\begin{tabular}{|c|c|c|c|c|c|c|}
\hline & \multicolumn{3}{|c|}{ Experimental } & \multicolumn{3}{|c|}{ Reference } \\
\hline & Baseline $(n=25)$ & $90 \mathrm{~d}(n=19)$ & $180 \mathrm{~d}(n=11)$ & Baseline $(n=33)$ & $90 \mathrm{~d}(n=27)$ & $180 \mathrm{~d}(n=20)$ \\
\hline $\mathrm{TAG}_{(\mathrm{mg} / \mathrm{dL})^{\dagger}}$ & $164 \pm 23$ & $143 \pm 17$ & $140 \pm 20$ & $129 \pm 10$ & $117 \pm 11$ & $141 \pm 22$ \\
\hline $\mathrm{TC}(\mathrm{mg} / \mathrm{dL})$ & $170 \pm 7$ & $178 \pm 9$ & $189 \pm 12$ & $175 \pm 8$ & $162 \pm 7$ & $170 \pm 11$ \\
\hline $\mathrm{LDLc}(\mathrm{mg} / \mathrm{dL})$ & $97 \pm 6$ & $105 \pm 8$ & $110 \pm 12$ & $103 \pm 6$ & $95 \pm 6$ & $99 \pm 10$ \\
\hline ApoB (mg/dL) & $80 \pm 4$ & $92 \pm 6$ & $85 \pm 10$ & $75 \pm 4$ & $75 \pm 4$ & $75 \pm 7$ \\
\hline $\operatorname{HDLc}(\mathrm{mg} / \mathrm{dL})$ & $47 \pm 2$ & $50 \pm 3$ & $54 \pm 4$ & $50 \pm 3$ & $49 \pm 2$ & $48 \pm 3$ \\
\hline ApoA-I (mg/dL) & $133 \pm 5$ & $132 \pm 6$ & $135 \pm 4$ & $133 \pm 4$ & $131 \pm 5$ & $127 \pm 4$ \\
\hline
\end{tabular}

${ }^{\dagger}$ Significant differences per time, according to a general lineal model for repeated measures. $P<0.05$ was considered significant. ApoA-I, apolipoprotein AI; apoB, apolipoprotein B; TC, cholesterol; HDLc, high-density lipoprotein cholesterol; LDLc, low-density lipoprotein cholesterol; TAG, triacylglycerols; TC, total cholesterol.

were extracted with hexane 3 times. The hexane phase was evaporated, and the fatty acids were identified and quantified after methylation by gas-liquid chromatography using a $60 \mathrm{~m}$ long capillary column (32 mm internal diameter and $20 \mathrm{~mm}$ film thickness) impregnated with SP 2330 FS (Supelco, Bellefonte, CA, USA). Fatty acid methyl esters from plasma lipids were obtained as previously reported [34]. Briefly, the hexane extracts of the total plasma and lipid fractions were dissolved into $2 \mathrm{~mL}$ methanol: benzene $(4: 1 \mathrm{v} / \mathrm{v})$. Methylation was performed at $100^{\circ} \mathrm{C}$ for $1 \mathrm{~h}$ by adding $200 \mu \mathrm{L}$ acetyl chloride. After cooling, $5 \mathrm{~mL}$ of $0.43 \mathrm{M} \mathrm{K}_{2} \mathrm{CO}_{3}$ was added to stop the reaction and neutralize the mixture. The tubes were then shaken and centrifuged. The benzene upper phase was dried under $\mathrm{N}_{2}$ and was resuspended with $100 \mu \mathrm{L}$ hexane.

2.4. Statistical Analysis. All data are presented as the mean \pm SEM. Prior to statistical analyses, all variables were checked for normality and homogeneous variance using the Kolmogorov-Smirnov and Levene tests, respectively. Variables that did not follow normality were logarithmically (apoA-I, dienes slope, TAC, tocopherol, total $\mathrm{CoQ}_{10}$, MMP9, sVCAM-1, eSelectin, tPAI-1, IL-6, and hsCRP) or inversely (HDLc, sICAM-1, and GR) transformed. To test for differences between the groups at baseline and each time point, a $t$-test for independent data was performed on the variables (or transformed variables) following a normal distribution for each group. A nonparametric Mann-Whitney $U$ test was performed for variables that did not follow a normal pattern (apoB, oxLDL, COMET tail moment, catalase, SOD, MCP-1, endothelin-1, TNF- $\alpha$, and MPO). For the data with a normal distribution and within each group, a one-way ANOVA and a posteriori Bonferroni tests were performed to evaluate the differences between feeding times. When the variables or transformed variables did not follow a normal pattern, Kruskal-Wallis and a posteriori Mann-Whitney $U$ tests were performed. To establish differences between groups and the interaction time $\times$ group, we used a general linear model of variance for repeated measures (GLM-RM) for patients who completed the study ( $n=14$ and $n=19$ for the experimental and reference groups, resp.). A value of $P<0.05$ was considered significant. The data analyses were performed using a statistical software package (SPSS for Windows, 15.0, 2005, SPSS Inc., Chicago, IL, USA).

\section{Results}

During the study period, the haematological parameters were controlled in all participants. No changes in white blood cells, coagulation indicators, or plasma electrolytes were observed (data not shown).

3.1. Plasma Lipids. Plasma concentrations of TAG, TC, apoB, HDLc, LDLc, and apoA-I were similar between both groups of intervention at all times. Feeding the two diets did not significantly modify TC, LDLc, apoB, HDLc, or apoA-I, but the effect of each diet tended to be different for LDLc $(P=0.088)$ and HDLc $(P=0.069)$ (Table 2$)$. In addition, the intervention significantly affected plasma TAG $(P=$ 0.042 ), especially in the experimental group, since plasma concentrations decreased after the intervention with the experimental diet but not after the reference diet $(P$ value for time per group interaction 0.074) (Table 2).

3.2. Oxidative Stress Biomarkers. When analysing diene formation during in vitro-induced LDL oxidation, the lag phase decreased after 90 days of feeding the experimental diet but returned to baseline conditions after 180 days. The lag phase was constant in subjects administered the reference product. However, the rate of in vitro-induced diene formation increased with time only in the reference group. LDL in vitroinduced hydroperoxide levels were lower in the experimental versus the reference group and this value did not change with dietary feeding in either group (Table 3 ). The oxLDL plasma concentrations were similar during the intervention in both groups (Table 3). There were no differences regarding lymphocyte DNA damage due to dietary intervention in either group, although the Comet Tail Moment was higher in the experimental group at baseline and throughout the interventional period (Table 3 ).

3.3. Antioxidant Defence System. Plasma TAC and retinol concentrations were unmodified by the two diets. However, the TAC values were lower in the experimental group (Figure 1). Plasma tocopherol levels had decreased in the reference group at 90 days but recovered to baseline levels after 180 days. The values did not change with the experimental diet. $\mathrm{CoQ}_{10}$, both oxidized and reduced, were lower 
TABLE 3: Blood oxidative stress parameters of frail adult and elderly patients fed exclusively by total enteral nutrition with the experimental and reference diets for $90 \mathrm{~d}$ and $180 \mathrm{~d}$.

\begin{tabular}{|c|c|c|c|c|c|c|}
\hline & \multicolumn{3}{|c|}{ Experimental } & \multicolumn{3}{|c|}{ Reference } \\
\hline & Baseline $(n=26)$ & $90 \mathrm{~d}(n=21)$ & $180 \mathrm{~d}(n=14)$ & Baseline $(n=28)$ & $90 \mathrm{~d}(n=24)$ & $180 \mathrm{~d}(n=19)$ \\
\hline \multicolumn{7}{|l|}{ LDL susceptibility to oxidation } \\
\hline Dienes lag Phase (min) & $78.1 \pm 3.4^{\mathrm{b}}$ & $58.1 \pm 3.0^{\mathrm{a}}$ & $71.5 \pm 4.0^{\mathrm{b}}$ & $70.5 \pm 3.0$ & $67.67 \pm 2.7$ & $70.6 \pm 4.6$ \\
\hline Dienes slope (mU OD/min) & $1.30 \pm 0.09$ & $1.56 \pm 0.16$ & $1.89 \pm 0.24$ & $1.26 \pm 0.10^{\mathrm{a}}$ & $1.28 \pm 0.12^{\mathrm{a}}$ & $1.82 \pm 0.16^{\mathrm{b}}$ \\
\hline LDL hydroperoxides* & $2.67 \pm 0.21$ & $3.66 \pm 0.34$ & $3.26 \pm 0.18$ & $4.56 \pm 0.31$ & $3.61 \pm 0.39$ & $4.15 \pm 0.31$ \\
\hline OxLDL (ng/mL) & $167.7 \pm 26.7$ & $166.7 \pm 25.2$ & $175.6 \pm 28.9$ & $126.2 \pm 12.5$ & $156.5 \pm 29.6$ & $129.9 \pm 14.1$ \\
\hline \multicolumn{7}{|l|}{ DNA damage } \\
\hline Comet head (\%) & $72.8 \pm 2.2$ & $76.8 \pm 2.1$ & $75.6 \pm 1.6$ & $75.45 \pm 2.0$ & $78.7 \pm 1.7$ & $79.8 \pm 1.8$ \\
\hline Comet tail (\%) & $24.4 \pm 1.6$ & $27.2 \pm 2.2$ & $23.2 \pm 2.1$ & $20.2 \pm 1.8$ & $24.5 \pm 2.1$ & $21.3 \pm 1.7$ \\
\hline Comet tail moment* & $0.53 \pm 0.05$ & $0.60 \pm 0.08$ & $0.51 \pm 0.07$ & $0.37 \pm 0.05^{\&}$ & $0.43 \pm 0.05$ & $0.37 \pm 0.04$ \\
\hline
\end{tabular}

${ }^{*}$ Significant difference between groups according to a general lineal model for repeated measures. ${ }^{\circledR}$ Significant differences between groups at each time point. Mean values within a row with unlike superscript letters are significantly different in each group. $P<0.05$ was considered significant. LDL hydroperoxide units are nmol/mg LDL protein. LDL, low-density lipoprotein; OD, optical density; oxLDL, oxidized low-density lipoprotein.

TABLE 4: Plasma cardiovascular and inflammatory biomarkers of frail adult and elderly patients fed exclusively by enteral nutrition with the experimental and reference diets for $90 \mathrm{~d}$ and $180 \mathrm{~d}$.

\begin{tabular}{|c|c|c|c|c|c|c|}
\hline & \multicolumn{3}{|c|}{ Experimental } & \multicolumn{3}{|c|}{ Reference } \\
\hline & Baseline $(n=26)$ & $90 \mathrm{~d}(n=21)$ & $180 \mathrm{~d}(n=14)$ & Baseline $(n=28)$ & $90 \mathrm{~d}(n=24)$ & $180 \mathrm{~d}(n=19)$ \\
\hline MMP-9 (ng/mL) & $156.6 \pm 20.5^{\mathrm{b}}$ & $112.6 \pm 17.7^{\mathrm{ab}}$ & $69.7 \pm 8.6^{\mathrm{a}}$ & $121.1 \pm 12.9^{\mathrm{b}}$ & $81.4 \pm 13.4^{\mathrm{a}}$ & $61.0 \pm 8.6^{\mathrm{a}}$ \\
\hline sVCAM-1 ( $\mu \mathrm{g} / \mathrm{mL})$ & $1.07 \pm 0.05$ & $1.13 \pm 0.08$ & $1.34 \pm 0.09$ & $1.07 \pm 0.08$ & $1.08 \pm 0.09$ & $1.06 \pm 0.10$ \\
\hline sICAM-1 (ng/mL) & $211.6 \pm 12.6$ & $214.3 \pm 12.8$ & $253.9 \pm 23.0$ & $199.6 \pm 9.59$ & $204.0 \pm 11.1$ & $189.6 \pm 7.8$ \\
\hline sE-Selectin (ng/mL) & $16.59 \pm 1.4$ & $16.8 \pm 1.3$ & $17.4 \pm 3.3$ & $16.23 \pm 1.2$ & $15.6 \pm 1.8$ & $16.6 \pm 1.7$ \\
\hline MCP-1 (pg/mL) & $202.0 \pm 16.2$ & $174.8 \pm 16.9$ & $161.6 \pm 16.5$ & $173.5 \pm 13.4$ & $144.6 \pm 8.8$ & $152.5 \pm 20.5$ \\
\hline Endothelin-1 (pmol/L) & $2.22 \pm 0.90$ & $1.66 \pm 0.94$ & $1.80 \pm 1.09$ & $2.07 \pm 0.50$ & $1.84 \pm 0.45^{\&}$ & $2.32 \pm 0.95^{\&}$ \\
\hline tPAI-1 $(\mathrm{ng} / \mathrm{mL})^{\gamma}$ & $10.32 \pm 2.26$ & $8.76 \pm 2.49$ & $5.08 \pm 1.41$ & $7.44 \pm 1.98$ & $8.01 \pm 2.30$ & $8.63 \pm 2.05$ \\
\hline MPO (ng/mL) & $21.76 \pm 4.1$ & $17.2 \pm 3.0$ & $14.3 \pm 1.7$ & $16.1 \pm 2.1$ & $16.7 \pm 3.9$ & $21.3 \pm 3.8$ \\
\hline $\mathrm{TNF} \alpha(\mathrm{ng} / \mathrm{mL})$ & $7.06 \pm 0.92$ & $7.29 \pm 1.69$ & $6.52 \pm 0.79$ & $5.60 \pm 0.61$ & $4.87 \pm 0.55$ & $4.72 \pm 0.55$ \\
\hline IL-6 (pg/mL) & $35.1 \pm 6.5$ & $40.0 \pm 8.6$ & $20.6 \pm 7.5$ & $35.8 \pm 11.7$ & $54.7 \pm 18.6$ & $40.2 \pm 19.4$ \\
\hline hsCRP (mg/L) & $2.03 \pm 0.42$ & $2.12 \pm 0.65$ & $0.98 \pm 0.32$ & $2.02 \pm 0.59$ & $1.88 \pm 0.60$ & $1.88 \pm 0.55$ \\
\hline
\end{tabular}

${ }^{8}$ Significant differences between groups at each time point. ${ }^{\gamma}$ Significant differences time per group according to a general lineal model for repeated measures. Mean values within a row with unlike superscript letters are significantly different in each group. $P<0.05$ was considered significant. IL-6, interleukin-6; MCP1, monocyte chemoattractant protein-1; MMP-9, metalloproteinase-9; MPO, myeloperoxidase; sICAM-1, soluble intercellular adhesion molecule-1; sVCAM-1, soluble vascular cell adhesion molecule-1; TNF- $\alpha$, tumour necrosis factor- $\alpha$; tPAI-1, total plasminogen activator inhibitor-1; hsCRP, high sensitive C reactive protein.

in the experimental group. The effect of enteral feeding was similar in both groups. Total- $\mathrm{CoQ}_{10}$ and reduced-CoQ $\mathrm{Q}_{10}$ were decreased at 90 and 180 days in the reference group. Only reduced-CoQ $\mathrm{Q}_{10}$ significantly decreased in the experimental group (Figure 1). Antioxidant GR, catalase, and SOD enzymatic activities were different between groups, and the effects of both enteral diets were significantly different for GPx, GR, and SOD (time $\times$ treatment effect). GPx, GR, and SOD activities decreased after administration of the experimental formula. Catalase activity did not change. GPx, GR, and catalase activities were unmodified in the reference group. SOD was increased at 90 and 180 days in the reference group (Figure 2).

3.4. Inflammatory and Cardiovascular Risk Biomarkers. The plasma levels of inflammatory and CVD risk biomarkers are presented in Table 4 . The levels of inflammatory biomarkers (TNF- $\alpha$, IL-6, and hsCRP) were similar in all volunteers at the beginning of the study and at 90 and 180 days. Regarding vascular function, endothelin-1 was lower in the experimental group at 90 and 180 days than in the reference group. Administration of both the experimental and reference formulae for 90 and 180 days significantly decreased the plasma concentrations of MMP-9. The effect on tPAI-1 was different between diets (time $\times$ treatment) (Table 4 ). tPAI-1 tended to decrease over time $(P=0.116)$ only in the experimental group.

3.5. Fatty Acids in Red Blood Cell Membranes. Table 5 presents the fatty acid profiles of RBC membranes. The linoleic acid (LA) and linolenic acid (LNA) percentages were different in both groups. LA was higher at 90 and 180 days, whereas LNA was lower at baseline and 90 days in the experimental than in the reference group. In addition, $n$ 3 LC-PUFAs tended to be higher $(P=0.053)$ after the experimental diet than after the reference diet (Table 5). 


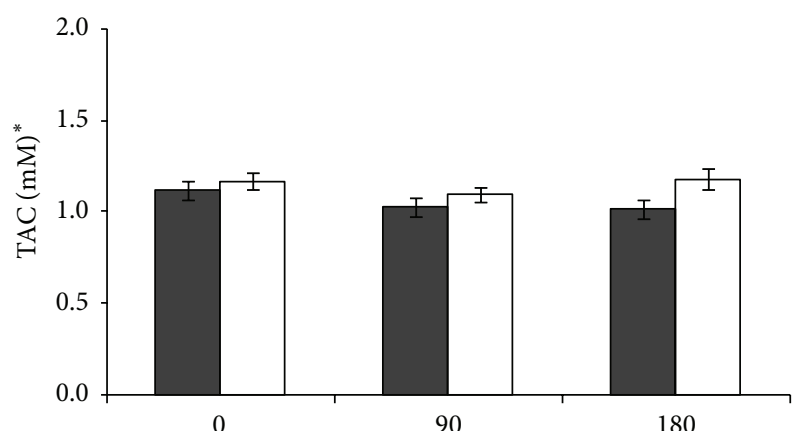

(d)

Experimental $\square$ Reference

(a)

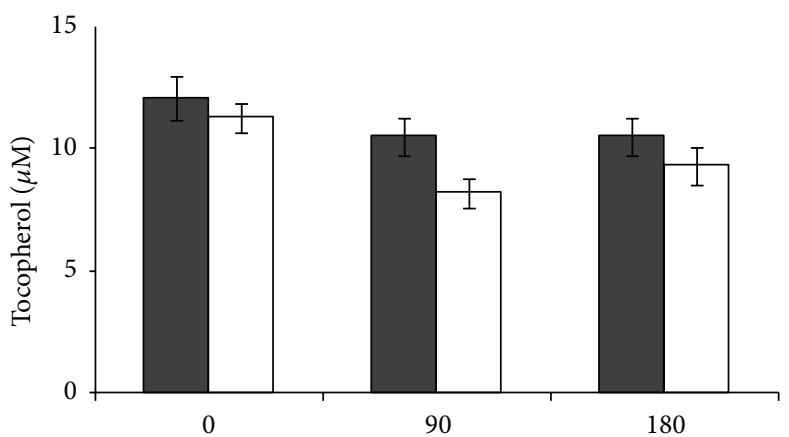

(d)

Experimental

$\square$ Reference

(c)

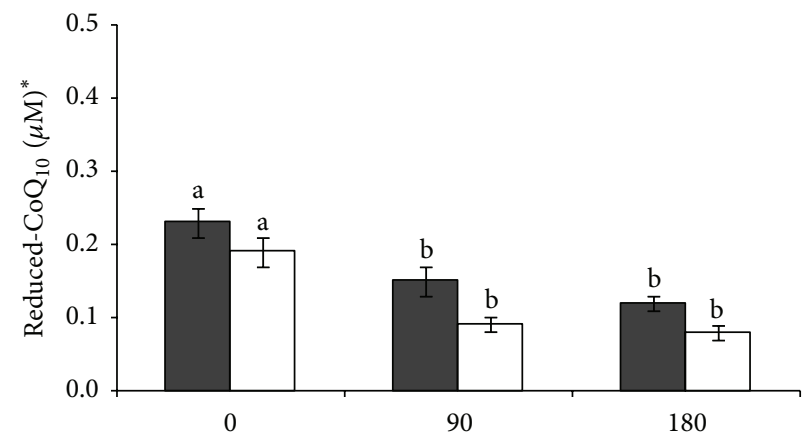

(d)

Experimental

$\square$ Reference

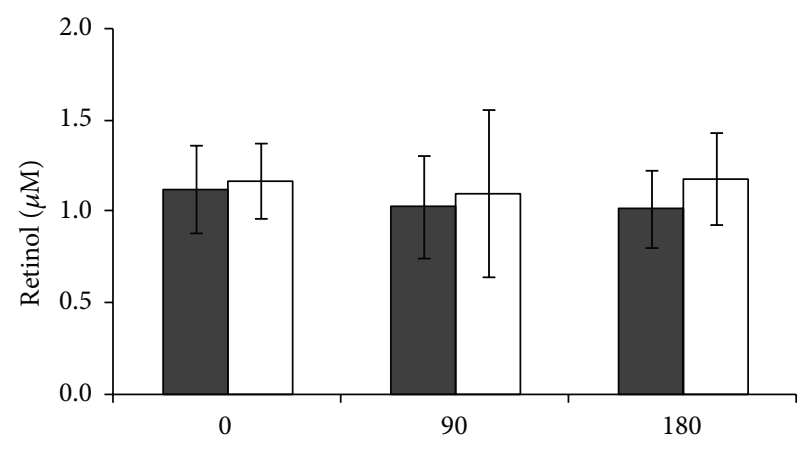

(d)

Experimental

$\square$ Reference

(b)

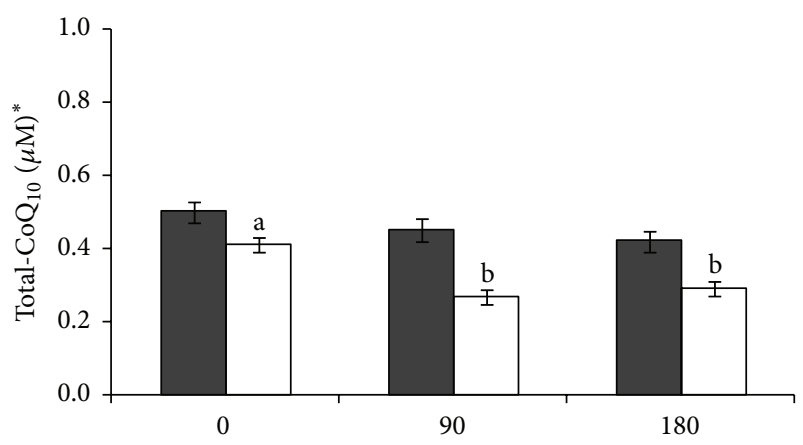

(d)

(d)

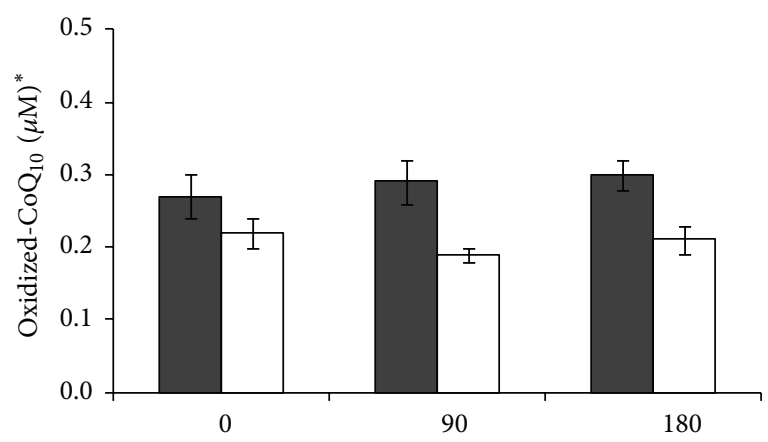

(d)

- Experimental

$\square$ Reference

(e)

(f)

FIGURE 1: Plasma nonenzymatic antioxidant defence system parameters (a) TAC, (b) retinol, (c) tocopherol, (d) total-CoQ 10 , (e) reduced$\mathrm{CoQ}_{10}$, and (f) oxidized-CoQ $\mathrm{Q}_{10}$ of frail adults and elderly patients fed exclusively by total enteral nutrition with the experimental and reference diets for $90 \mathrm{~d}$ and $180 \mathrm{~d}$. *Significant differences between groups according to a general lineal model for repeated measures. Different letters indicate significant differences between times within each group. $P<0.05$ was considered significant. TAC, total antioxidant capacity.

The effect of both enteral diets on arachidonic acid (AA) and docosapentaenoic acid (DPA) were different in both groups. The administration of the experimental diet decreased AA after 90 days and increased DPA after 180 days. AA was unmodified and EPA decreased with the reference diet. Indeed, the reference diet increased palmitic acid at 90 days.

\section{Discussion}

The present study evaluated the effect of an $n-3$ LC-PUFAenriched enteral formula on oxidative stress, the antioxidant defence system, inflammatory and CVD risk biomarkers, and the RBC fatty acid profile in fragile adults and elderly 


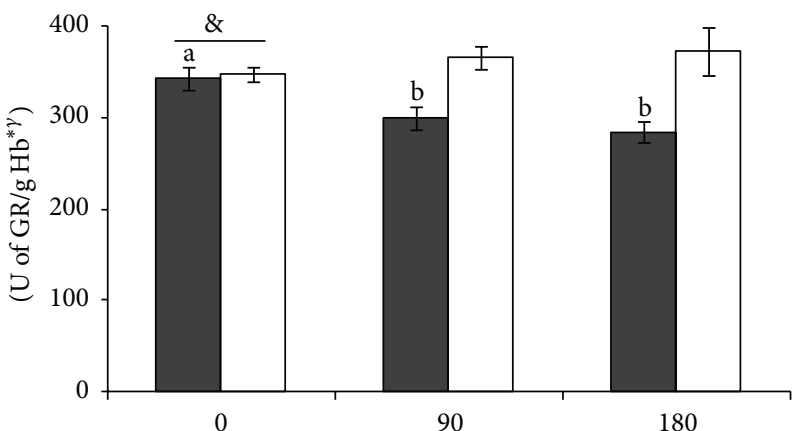

(d)

Experimental $\square$ Reference

(a)

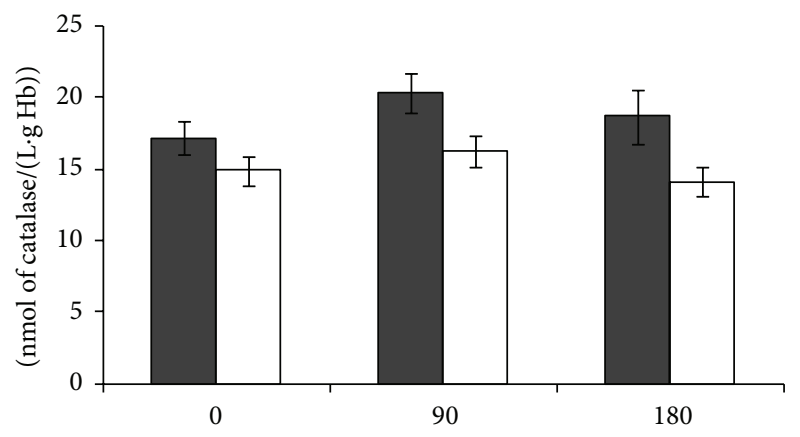

(d)

Experimental $\square$ Reference

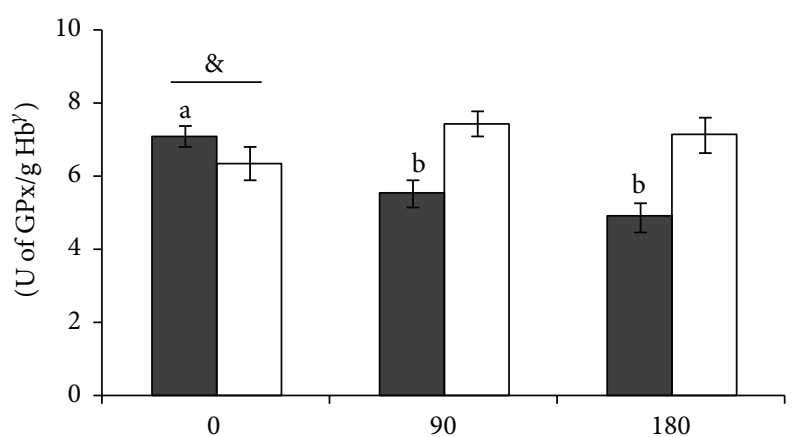

(d)

Experimental $\square$ Reference

(b)

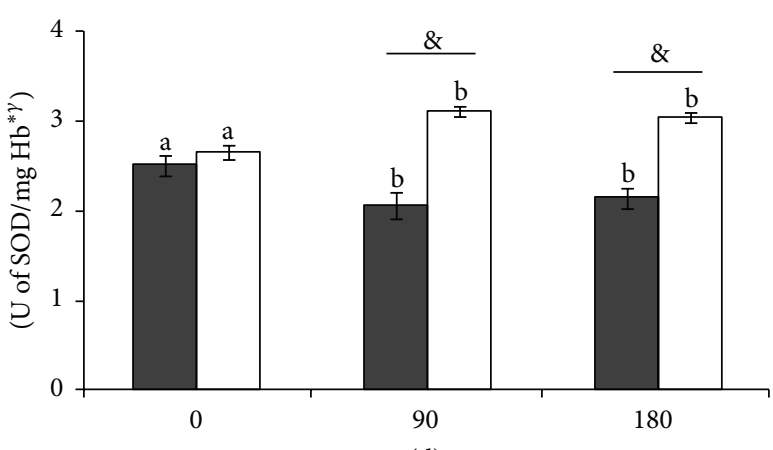

(d)
Experimental $\square$ Reference

(c)

(d)

Figure 2: Red blood cell enzymatic antioxidant activities (a) GR, (b) GPx, (c) catalase, and (d) SOD of frail adult and elderly patients fed exclusively by total enteral nutrition with the experimental and reference diets for $90 \mathrm{~d}$ and $180 \mathrm{~d}$. *Significant differences between groups according to GLM-RM. ${ }^{8}$ Significant differences between groups at each time point. ${ }^{\gamma}$ Significant differences in the interaction time $\times$ group according to GLM-RM. Different letters indicate significant differences between times within each group. $P<0.05$ was considered significant. GLM-RM, general lineal model for repeated measures; GPx, glutathione peroxidase; GR, glutathione reductase; SOD, superoxide dismutase.

TABLE 5: Fatty acids in red blood cell membranes of frail adult and elderly patients fed exclusively by total enteral nutrition with the experimental and reference diets for $90 \mathrm{~d}$ and $180 \mathrm{~d}$.

\begin{tabular}{|c|c|c|c|c|c|c|}
\hline \multirow{2}{*}{$(\%)$} & \multicolumn{3}{|c|}{ Experimental } & \multicolumn{3}{|c|}{ Reference } \\
\hline & Baseline $(n=25)$ & $90 \mathrm{~d}(n=21)$ & $180 \mathrm{~d}(n=14)$ & Baseline $(n=27)$ & $90 \mathrm{~d}(n=24)$ & $180 \mathrm{~d}(n=18)$ \\
\hline Palmitic acid & $23.3 \pm 0.4$ & $24.6 \pm 0.4$ & $23.6 \pm 0.5$ & $22.3 \pm 0.8^{\mathrm{a}}$ & $23.5 \pm 0.3^{\mathrm{b}}$ & $23.7 \pm 0.3^{\mathrm{ab}}$ \\
\hline Stearic acid & $13.6 \pm 0.4$ & $13.6 \pm 0.3$ & $13.9 \pm 0.2$ & $13.6 \pm 0.3$ & $13.4 \pm 0.3$ & $13.8 \pm 0.4$ \\
\hline Oleic acid & $17.6 \pm 0.5$ & $17.8 \pm 0.5$ & $17.5 \pm 0.4$ & $18.1 \pm 0.4$ & $17.9 \pm 0.4$ & $18.2 \pm 0.6$ \\
\hline $\mathrm{LA}^{*}$ & $8.8 \pm 0.3$ & $9.6 \pm 0.4$ & $9.4 \pm 0.4$ & $8.6 \pm 0.3$ & $8.2 \pm 0.3^{8}$ & $8.2 \pm 0.2^{\&}$ \\
\hline LNA $^{*}$ & $0.24 \pm 0.01$ & $0.22 \pm 0.01$ & $0.26 \pm 0.02$ & $0.3 \pm 0.02^{\&}$ & $0.29 \pm 0.02^{\&}$ & $0.27 \pm 0.01$ \\
\hline AA & $14.0 \pm 0.4^{\mathrm{b}}$ & $12.3 \pm 0.5^{\mathrm{a}}$ & $12.3 \pm 0.6^{\mathrm{ab}}$ & $14.6 \pm 0.4$ & $13.5 \pm 0.5$ & $13.7 \pm 0.4$ \\
\hline EPA & $0.59 \pm 0.04$ & $0.55 \pm 0.04$ & $0.58 \pm 0.03$ & $0.71 \pm 0.05^{\mathrm{b}}$ & $0.53 \pm 0.04^{\mathrm{a}}$ & $0.49 \pm 0.04^{\mathrm{a} \gamma}$ \\
\hline $\mathrm{DPA}^{\gamma}$ & $1.5 \pm 0.1^{\mathrm{a}}$ & $1.7 \pm 0.1^{\mathrm{a}}$ & $2.2 \pm 0.1^{\mathrm{b}}$ & $1.9 \pm 0.4$ & $1.8 \pm 0.1$ & $1.7 \pm 0.1$ \\
\hline DHA & $4.0 \pm 0.3$ & $3.6 \pm 0.2$ & $3.6 \pm 0.2$ & $3.4 \pm 0.3$ & $2.8 \pm 0.2^{\gamma}$ & $3.0 \pm 0.2$ \\
\hline$n-3$ LC-PUFA & $6.0 \pm 0.3$ & $5.8 \pm 0.3$ & $6.4 \pm 0.2$ & $6.0 \pm 0.5$ & $5.2 \pm 0.21$ & $5.1 \pm 0.3$ \\
\hline
\end{tabular}

${ }^{*}$ Significant difference between groups according to a general lineal model for repeated measures. ${ }^{\gamma}$ Significant differences between groups at each time point. Mean values within a row with unlike superscript letters are significantly different in each group. ${ }^{\&}$ Significant differences between groups at each time point. $P<0.05$ was considered significant. AA, arachidonic acid; DHA, docosahexaenoic acid; DPA, docosapentaenoic acid; EPA, eicosapentaenoic acid; LA, linoleic acid; LNA linolenic acid; $n$-3 LC-PUFA, omega-3 long chain polyunsaturated fatty acids. 
subjects to ascertain its suitability for nutritional treatment. The main results of the present study were that the enteral administration of $n$ - 3 LC-PUFAs tended to increase the RBC levels without an increase in LDL susceptibility to oxidation or DNA integrity. In addition, the plasma antioxidant capacity was maintained throughout the intervention. Reduced$\mathrm{CoQ}_{10}$ and antioxidant enzymes (GPx, GR, and SOD) activities were decreased in the volunteers fed the $n$ - 3 LC-PUFAenriched diet. Moreover, TAG and the CDV biomarker tPAI1 decreased only after the intake of the $n$-3 LC-PUFA diet whereas MMP-9 decreased in both intervention groups. It is well documented that modest consumption of fish or fish oil supplementation positively affects cardiac haemodynamics [35], which could partially account for the clinical benefits of fish or fish oil intake, including a lower risk of cardiac death [36], cerebrovascular disease [37], ischaemic stroke, heart failure [38], cognitive decline [39], and nonfatal coronary events [40]. In addition, EPA and DHA-derived molecules exert anti-inflammatory and inflammatory-resolving effects (opposite of AA) and lower the production of inflammatory cytokines following reduced activation of proinflammatory transcription factors, such as NF- $\kappa \mathrm{B}[41,42]$. Therefore, increased $n$-3 LC-PUFAs in membranes may be helpful for a variety of acute and chronic inflammatory processes that occur during ageing [43]. On the other hand, a well-known beneficial effect of $n$-3 LC-PUFAs is the optimization of plasma TAG metabolism [44], a well-known cardiovascular risk biomarker. We have previously reported that feeding enteral formula enriched in EPA and DHA lowers plasma TAG without affecting insulin resistance [24]. In the present study, we have selected patients over 50 years old participating in that study [24] and we have also observed a significant effect on plasma TAG. No other plasma lipid was affected by the enteral feeding with any diet as previously reported [24].

However, the high degree of unsaturation of $n-3$ PUFA makes these fatty acids highly susceptible to oxidation, which may render them harmful, leading to dysfunctional membranes and to increased oxidative molecules that trigger inflammatory responses $[4,23]$. In the present study, we observed that LDL particles were not more susceptible to in vitro oxidation after the administration of $n-3$ PUFAs. In addition, we did not observe increased in vivo plasma oxLDL levels or modifications on blood lymphocyte DNA oxidative damage. Indeed, serum TAC was also unmodified reflecting that the oxidative status was not impaired following the ingestion of $n$-3 LC-PUFAs for 6 months. Some authors have reported that $n-3$ LC-PUFA administration increased LDL susceptibility to oxidation $[45,46]$. In accordance with our results, some authors have reported that fish oil supplementation does not increase the overall oxidation of LDL ex vivo [47] or the systemic oxidative stress [48, 49]. Furthermore, the increased susceptibility of LDL to in vitro oxidation observed in haemodialysis patients can be reduced by supplementation with fish oil containing vitamin $\mathrm{E}$ as an antioxidant [50]. Other authors have observed that supplementation of dairy products with $n-3$ LC-PUFA and an adequate amount of vitamin $\mathrm{E}$ decreases cardiovascular risk factors without causing additional oxidative DNA damage [51]. This may explain the oxidative stress stability of our volunteers fed the $n$-3 LC-PUFA-enriched diet, which is well stabilized against peroxidation ( $1 \mathrm{mg}$ of $\alpha$-tocoferol/g of $n-3$ PUFA, Table 1).

This oxidative stress stability may require lower antioxidant defences and that may be the reason for the lower induction of RBC antioxidant defence found in the present study. Although antioxidant enzyme activities decreased after n-3 LC-PUFA administration, this does not necessarily mean an impaired antioxidant capacity in RBCs but perhaps a compensation of activity in subjects with a lower risk of oxidative stress and with a frailty metabolism. The effect of n-3 LC-PUFA administration on RBC antioxidant enzymatic activity is not clear because some authors have described induction [52-55] and others have observed a null effect $[49,56,57]$ depending on the administration dosage and duration. In addition, the decrease in plasma reduced-CoQ $\mathrm{Q}_{10}$ may be caused by a lower GR activity that is not able to regenerate from the oxidised form. On the other hand, we cannot explain the lower plasma concentrations of total and reduced $\mathrm{CoQ}_{10}$ after feeding the reference diet, but this fact may influence the higher rate of LDL oxidation found in this group of patients at the end of the intervention.

Proinflammatory molecules may be implicated in the initiation, progression, and plaque instability of the atherosclerotic process. MMPs are a large family of proteolytic enzymes involved in the remodelling of several components of the extracellular matrix that play a role in many physiological and pathological processes $[58,59]$. The beneficial effect of $n-3$ LC-PUFAs lowering MMP-9 expression and activity has been previously reported [60-62], although other authors have reported no effect $[63,64]$. Our data indicate that EN with both formulae reduced plasma MMP-9 levels. Therefore, we cannot attribute the beneficial effect to $n$-3 LC-PUFAs but to an adequate supply of nutrients because the reference product did not provide these ingredients and caused a similar effect.

We did not observe a positive effect of the $n-3$ LCPUFA formula on plasma levels of any other inflammatory biomarker. The effects of $n-3$ LC-PUFAs are not clear. A recent meta-analysis provided consistent evidence that marine-derived $n$ - 3 PUFAs supplementation had a significant lowering effect on fasting blood levels of CRP, IL-6, and TNF- $\alpha$ in subjects with chronic nonautoimmune disease and healthy subjects. Those authors found a significant negative linear relationship between the duration and effect size of marine-derived $n$-3 PUFA supplementation on fasting blood levels of TNF- $\alpha$ and IL-6 [65]. In the present study, the plasma levels of hsCRP, IL-6, and TNF- $\alpha$ were relatively low at baseline, which could explain why $n$-3 LC-PUFAs had no observable effects later. With regard to CVD risk biomarkers, some studies report no influence on sICAM-1 after a high dose of $n$-3 LC-PUFAs $[66,67]$ but an increase in sVCAM-1 [67]. However, other studies have reported that n-3 LC-PUFAs may decrease these endothelial-derived molecules [68, 69]. In addition, Adkins and Kelley [70] reviewed the underlying cardioprotective mechanisms of $n-3$ LC-PUFAs, including the inhibition of monocyte infiltration due to decreased sVCAM-1, sICAM-1, and MCP-1 (another molecule that was not modified after $n$-3 LC-PUFAs feeding). In accordance with our results, some authors have described 
that $n$-3 LC-PUFA supplementation had no effect on the plasma levels of MPO in healthy adults [71].

Endothelin-1 is a potent vasoconstrictor peptide and accepted marker of increased oxidative stress secreted by endothelial cells. As previously described [72], we did not observe any effect of the inclusion of EPA and DHA on endothelin-1 in our patients. We observed that after 90 and 180 days of enteral feeding, the subjects receiving the $n-3$ LC PUFA-enriched formula had lower levels of circulating endothelin-1 than those in the reference group, which may be an indicator of a better vascular status. In addition, we identified differences between both groups for tPAI-1 plasma levels. The $n$-3 LC PUFA-enriched diet tended to diminish tPAI-1 plasma levels, and the reference diet did not cause this effect. tPAI-1 is associated with CVD risk and has an important role in the development of vascular thrombosis [73]. The beneficial effect of $n-3$ LC-PUFAs on tPAI levels has been previously reported [74]. We speculate that the frailty of patients included in the present study makes it difficult to find positive effects with the current doses and intervention times. Therefore, studies with longer intervention periods might be interesting to ascertain whether the formula has an impact on these vascular functional biomarkers, morbidity, and mortality.

In conclusion, the present work indicates that exclusive feeding with an enteral formula enriched with EPA and DHA does not impair the oxidative status of fragile adults and elderly patients and could have a positive effect on some inflammatory biomarkers demonstrating a nutritional tool that may help in the prevention of cardiovascular age-related complications without adverse effects.

\section{Conflict of Interests}

Maria D. Mesa, Josune Olza, Carolina Gonzalez-Anton Concepcion M. Aguilera, Azahara I. Ruperez, Rosario MorenoTorres, and Antonio Perez de la Cruz declare no conflict of interests. Mrs. Africa Jiménez is a member of the Research and Development Department of Vegenat (the company that funded the present study) and was involved in the development of the T-Diet Plus product. Professor Angel Gil has no contractual relationship with Vegenat; however, he participated in the product design as part of a research contract between the University of Granada Foundation and Vegenat.

\section{Acknowledgments}

This project was funded by an Enterprise-University project between the University of Granada Foundation and Vegenat S. A. (FEUGR Project no. 2559). The authors thank the patients and the Institution for participating in this study.

\section{References}

[1] A. Kalache and A. Gatti, "Active ageing: a policy framework," Advances in Gerontology, vol. 11, pp. 7-18, 2003.

[2] E. Ylimaki, O. Kanste, H. Heikkinen, R. Bloigu, and H. Kyngas, "The effects of a counselling intervention on lifestyle change in people at risk of cardiovascular disease," European Journal of Cardiovascular Nursing, vol. 14, no. 2, pp. 153-161, 2015.

[3] S. Vasto, G. Candore, C. R. Balistreri et al., "Inflammatory networks in ageing, age-related diseases and longevity," Mechanisms of Ageing and Development, vol. 128, no. 1, pp. 83-91, 2007.

[4] J. A. Leopold and J. Loscalzo, "Oxidative risk for atherothrombotic cardiovascular disease," Free Radical Biology and Medicine, vol. 47, no. 12, pp. 1673-1706, 2009.

[5] K. M. Desai, T. Chang, H. Wang et al., "Oxidative stress and aging: is methylglyoxal the hidden enemy?" Canadian Journal of Physiology and Pharmacology, vol. 88, no. 3, pp. 273-284, 2010.

[6] A. J. Donato, R. G. Morgan, A. E. Walker, and L. A. Lesniewski, "Cellular and molecular biology of aging endothelial cells," Journal of Molecular and Cellular Cardiology, 2015.

[7] A. B. Salmon, A. Richardson, and V. I. Pérez, "Update on the oxidative stress theory of aging: does oxidative stress play a role in aging or healthy aging?" Free Radical Biology and Medicine, vol. 48, no. 5, pp. 642-655, 2010.

[8] M. De La Fuente and J. Miquel, "An update of the oxidationinflammation theory of aging: the involvement of the immune system in oxi-inflamm-aging," Current Pharmaceutical Design, vol. 15, no. 26, pp. 3003-3026, 2009.

[9] J. Mulero, P. Zafrilla, and A. Martinez-Cacha, "Oxidative stress, frailty and cognitive decline," The Journal of Nutrition, Health \& Aging, vol. 15, no. 9, pp. 756-760, 2011.

[10] R. S. Tupe, A. G. Diwan, V. D. Mittal, P. S. Narayanam, and K. B. Mahajan, "Association of plasma proteins at multiple stages of glycation and antioxidant status with erythrocyte oxidative stress in patients with type 2 diabetes," British Journal of Biomedical Science, vol. 71, no. 3, pp. 93-138, 2014.

[11] E. Toussaint, A. Van Gossum, A. Ballarin, and M. Arvanitakis, "Enteral access in adults," Clinical Nutrition, vol. 34, no. 3, pp. 350-358, 2015.

[12] I. Stange, K. Poeschl, P. Stehle, C. C. Sieber, and D. Volkert, "Screening for malnutrition in nursing home residents: comparison of different risk markers and their association to functional impairment," Journal of Nutrition, Health and Aging, vol. 17, no. 4, pp. 357-363, 2013.

[13] C. C. Roy, L. Bouthillier, E. Seidman, and É. Levy, "New lipids in enteral feeding," Current Opinion in Clinical Nutrition and Metabolic Care, vol. 7, no. 2, pp. 117-122, 2004.

[14] E. A. Miles and P. C. Calder, "Fatty acids, lipid emulsions and the immune and inflammatory systems," World Review of Nutrition and Dietetics, vol. 112, pp. 17-30, 2015.

[15] M. D. Mesa, C. M. Aguilera, and A. Gil, "Importance of lipids in the nutritional treatment of inflammatory diseases," Nutrición Hospitalaria, vol. 21, pp. 30-43, 2006.

[16] P. C. Calder, "The role of marine omega-3 (n-3) fatty acids in inflammatory processes, atherosclerosis and plaque stability," Molecular Nutrition and Food Research, vol. 56, no. 7, pp. 10731080, 2012.

[17] H. Fares, C. J. Lavie, J. J. DiNicolantonio, J. H. O'Keefe, and R. V. Milani, "Omega-3 fatty acids: a growing ocean of choices.", Current Atherosclerosis Reports, vol. 16, no. 2, article 389, 2014.

[18] O. D. Rangel-Huerta, C. M. Aguilera, M. D. Mesa, and A. Gil, "Omega-3 long-chain polyunsaturated fatty acids supplementation on inflammatory biomakers: a systematic review of randomised clinical trials," British Journal of Nutrition, vol. 107, no. 2, pp. S159-S170, 2012.

[19] D. B. Jump, C. M. Depner, and S. Tripathy, "Omega-3 fatty acid supplementation and cardiovascular disease," Journal of Lipid Research, vol. 53, no. 12, pp. 2525-2545, 2012. 
[20] P. Flachs, M. Rossmeisl, M. Bryhn, and J. Kopecky, "Cellular and molecular effects of $n-3$ polyunsaturated fatty acids on adipose tissue biology and metabolism," Clinical Science, vol. 116, no. 1, pp. 1-16, 2009.

[21] FAO, "Fats and fatty acids in human nutrition: report of an expert consultation," FAO Food and Nutrition Paper 91, FAO, Rome, Italy, 2010.

[22] C. Enzenbach, J. Kröger, V. Zietemann et al., "Erythrocyte membrane phospholipid polyunsaturated fatty acids are related to plasma C-reactive protein and adiponectin in middle-aged German women and men," European Journal of Nutrition, vol. 50, no. 8, pp. 625-636, 2011.

[23] A. Catalá, "A synopsis of the process of lipid peroxidation since the discovery of the essential fatty acids," Biochemical and Biophysical Research Communications, vol. 399, no. 3, pp. 318$323,2010$.

[24] J. Olza, M. D. Mesa, C. M. Aguilera et al., "Influence of an eicosapentaenoic and docosahexaenoic acid-enriched enteral nutrition formula on plasma fatty acid composition and biomarkers of insulin resistance in the elderly," Clinical Nutrition, vol. 29, no. 1, pp. 31-37, 2010.

[25] B. H. Chung, T. Wilkinson, J. C. Geer, and J. P. Segrest, "Preparative and quantitative isolation of plasma lipoproteins: rapid, single discontinuous density gradient ultracentrifugation in a vertical rotor," Journal of Lipid Research, vol. 21, no. 3, pp. 284-291, 1980.

[26] M. D. Mesa, J. M. Silván, J. Olza, Á. Gil, and M. D. del Castillo, "Antioxidant properties of soy protein-fructooligosaccharide glycation systems and its hydrolyzates," Food Research International, vol. 41, no. 6, pp. 606-615, 2008.

[27] A. R. Collins, M. Dušinská, C. M. Gedik, and R. Štětina, "Oxidative damage to DNA: do we have a reliable biomarker?" Environmental Health Perspectives, vol. 104, no. 3, pp. 465-469, 1996.

[28] M. Battino, L. Leone, and S. Bompadre, "igh-performance liquid chromatography-EC assay of mitochondrial coenzyme Q9, coenzyme Q9H2, coenzyme Q10, coenzyme Q10H2, and vitamin E with a simplified on-line solid-phase extraction," Methods in Enzymology, vol. 378, pp. 156-162, 2004.

[29] D. L. Drabkin, “The standardization of hemoglobin measurement," The American Journal of the Medical Sciences, vol. 215, article 110, 1948.

[30] H. Aebi, "Catalase in vitro," Methods in Enzymology, vol. 105, pp. 121-126, 1984.

[31] J. M. McCord and I. Fridovich, "The utility of superoxide dismutase in studying free radical reactions. I. Radicals generated by the interaction of sulfite, dimethyl sulfoxide, and oxygen," The Journal of Biological Chemistry, vol. 244, no. 22, pp. 6056-6063, 1969.

[32] I. Carlberg and B. Mannervik, "Glutathione reductase," Methods in Enzymology, vol. 113, pp. 484-490, 1985.

[33] L. Flohe and W. A. Günzler, "Assays of glutathione peroxidase," Methods in Enzymology, vol. 105, pp. 114-121, 1984.

[34] G. Lepage and C. C. Roy, "Direct transesterification of all classes of lipids in a one-step reaction," The Journal of Lipid Research, vol. 27, no. 1, pp. 114-120, 1986.

[35] D. Mozaffarian and J. H. Y. Wu, “(n-3) Fatty acids and cardiovascular health: are effects of EPA and DHA shared or complementary?" Journal of Nutrition, vol. 142, no. 3, pp. 614S625S, 2012.
[36] L. M. Kimmig and D. G. Karalis, "Do omega-3 polyunsaturated fatty acids prevent cardiovascular disease? A review of the randomized clinical trials," Lipid Insights, vol. 6, pp. 13-20, 2013.

[37] R. Chowdhury, S. Stevens, D. Gorman et al., "Association between fish consumption, long chain omega 3 fatty acids, and risk of cerebrovascular disease: systematic review and metaanalysis," British Medical Journal, vol. 345, no. 7881, Article ID e6698, 2012.

[38] D. Mozaffarian and J. H. Y. Wu, "Omega-3 fatty acids and cardiovascular disease: effects on risk factors, molecular pathways, and clinical events," Journal of the American College of Cardiology, vol. 58, no. 20, pp. 2047-2067, 2011.

[39] T. Cederholm, N. Salem Jr., and J. Palmblad, “ $\omega$-3 fatty acids in the prevention of cognitive decline in humans," Advances in Nutrition, vol. 4, no. 6, pp. 672-676, 2013.

[40] D. Mozaffarian, "Fish, n-3 fatty acids, and cardiovascular haemodynamics," Journal of Cardiovascular Medicine, vol. 8, supplement 1, pp. S23-S26, 2007.

[41] P. Singer, H. Shapiro, M. Theilla, R. Anbar, J. Singer, and J. Cohen, "Anti-inflammatory properties of omega-3 fatty acids in critical illness: novel mechanisms and an integrative perspective," Intensive Care Medicine, vol. 34, no. 9, pp. 1580-1592, 2008.

[42] P. C. Calder, "N-3 Fatty acids, inflammation and immunity: new mechanisms to explain old actions," Proceedings of the Nutrition Society, vol. 72, no. 3, pp. 326-336, 2013.

[43] C. I. F. Janssen and A. J. Kiliaan, "Long-chain polyunsaturated fatty acids (LCPUFA) from genesis to senescence: the influence of LCPUFA on neural development, aging, and neurodegeneration," Progress in Lipid Research, vol. 53, no. 1, pp. 1-17, 2014.

[44] H. M. Roche and M. J. Gibney, "Effect of long-chain n-3 polyunsaturated fatty acids on fasting and postprandial triacylglycerol metabolism," The American Journal of Clinical Nutrition, vol. 71, no. 1, supplement, pp. 232S-237S, 2000.

[45] C. Ramírez-Tortosa, J. M. López-Pedrosa, A. Suarez, E. Ros, J. Mataix, and A. Gil, "Olive oil- and fish oil-enriched diets modify plasma lipids and susceptibility of LDL to oxidative modification in free-living male patients with peripheral vascular disease: the Spanish Nutrition Study," British Journal of Nutrition, vol. 82, no. 1, pp. 31-39, 1999.

[46] S. Egert, V. Somoza, F. Kannenberg et al., "Influence of three rapeseed oil-rich diets, fortified with alpha-linolenic acid, eicosapentaenoic acid or docosahexaenoic acid on the composition and oxidizability of low-density lipoproteins: results of a controlled study in healthy volunteers," European Journal of Clinical Nutrition, vol. 61, no. 3, pp. 314-325, 2007.

[47] J. V. Higdon, S. H. Du, Y. S. Lee, T. Wu, and R. C. Wander, "Supplementation of postmenopausal women with fish oil does not increase overall oxidation of LDL ex vivo compared to dietary oils rich in oleate and linoleate," Journal of Lipid Research, vol. 42, no. 3, pp. 407-418, 2001.

[48] D. Tousoulis, N. Papageorgiou, C. Antoniades et al., "Acute effects of different types of oil consumption on endothelial function, oxidative stress status and vascular inflammation in healthy volunteers," British Journal of Nutrition, vol. 103, no. 1, pp. 43-49, 2010.

[49] I. Ottestad, G. Vogt, K. Retterstøl et al., "Oxidised fish oil does not influence established markers of oxidative stress in healthy human subjects: a randomised controlled trial," British Journal of Nutrition, vol. 108, no. 2, pp. 315-326, 2012.

[50] O. Panzetta, L. Cominacini, U. Garbin et al., "Increased susceptibility of LDL to in vitro oxidation in patients on maintenance 
hemodialysis: effects of fish oil and vitamin E administration," Clinical Nephrology, vol. 44, no. 5, pp. 303-309, 1995.

[51] C. Dawczynski, L. Martin, A. Wagner, and G. Jahreis, "n-3 LCPUFA-enriched dairy products are able to reduce cardiovascular risk factors: a double-blind, cross-over study," Clinical Nutrition, vol. 29, no. 5, pp. 592-599, 2010.

[52] N. Lasztity, J. Hamvas, L. Biró et al., "Effect of enterally administered n-3 polyunsaturated fatty acids in acute pancreatitisa prospective randomized clinical trial," Clinical Nutrition, vol. 24, no. 2, pp. 198-205, 2005.

[53] H. Tayyebi-Khosroshahi, J. Houshyar, A. Tabrizi, A.-M. Vatankhah, N. R. Zonouz, and R. Dehghan-Hesari, "Effect of omega-3 fatty acid on oxidative stress in patients on hemodialysis," Iranian Journal of Kidney Diseases, vol. 4, no. 4, pp. 322-326, 2010.

[54] O. Firuzi, N. Shakibazad, H. Amoozgar et al., "Effects of omega3 polyunsaturated fatty acids on heart function and oxidative stress biomarkers in pediatric patients with dilated cardiomyopathy," International Cardiovascular Research Journal, vol. 7, no. 1, pp. 8-14, 2013.

[55] L. Lluís, N. Taltavull, M. Muñoz-Cortés et al., "Protective effect of the omega-3 polyunsaturated fatty acids: eicosapentaenoic acid/docosahexaenoic acid 1:1 ratio on cardiovascular disease risk markers in rats," Lipids in Health and Disease, vol. 12, article 140, 2013.

[56] G. M. Smith and A. Isopenko, "Effect of doses of protected polyunsaturated fatty acids on indicators of selenium status of sheep," Research in Veterinary Science, vol. 62, no. 1, pp. 81-82, 1997.

[57] M. Iraz, H. Erdogan, B. Ozyurt, F. Ozugurlu, S. Ozgocmen, and E. Fadillioglu, "Brief communication: omega-3 essential fatty acid supplementation and erythrocyte oxidant/antioxidant status in rats," Annals of Clinical and Laboratory Science, vol. 35, no. 2, pp. 169-173, 2005.

[58] J. Hu, P. E. Van den Steen, Q.-X. A. Sang, and G. Opdenakker, "Matrix metalloproteinase inhibitors as therapy for inflammatory and vascular diseases," Nature Reviews Drug Discovery, vol. 6, no. 6, pp. 480-498, 2007.

[59] C. Busti, E. Falcinelli, S. Momi, and P. Gresele, "Matrix metalloproteinases and peripheral arterial disease," Internal and Emergency Medicine, vol. 5, no. 1, pp. 13-25, 2010.

[60] L. Shinto, G. Marracci, L. Bumgarner, and V. Yadav, "The effects of omega-3 fatty acids on matrix metalloproteinase9 production and cell migration in human immune cells: implications for multiple sclerosis," Autoimmune Diseases, vol. 2011, Article ID 134592, 6 pages, 2011.

[61] G. Derosa, A. F. G. Cicero, E. Fogari et al., "Effects of n-3 PUFAs on postprandial variation of metalloproteinases, and inflammatory and insulin resistance parameters in dyslipidemic patients: evaluation with euglycemic clamp and oral fat load," Journal of Clinical Lipidology, vol. 6, no. 6, pp. 553-564, 2012.

[62] T. Chitranjali, P. Anoop Chandran, and G. Muraleedhara Kurup, "Omega-3 fatty acid concentrate from Dunaliella salina possesses anti-inflammatory properties including blockade of NF- $\kappa \mathrm{B}$ nuclear translocation," Immunopharmacology and Immunotoxicology, vol. 37, no. 1, pp. 81-89, 2015.

[63] J. Vogt, V. L. Andersen, A. Andreasen, T. Obel, J. H. Christensen, and E. B. Schmidt, "Serum concentrations of matrix metalloproteinase-9, tissue inhibitor of matrix metalloproteinase-1 and alpha2-macroglobulin in healthy subjects after supplementation with different doses of marine n-3 fatty acids," Cellular and Molecular Biology, vol. 56, no. 1, pp. 102-109, 2010.
[64] E. B. Furenes, I. Seljeflot, S. Solheim, E. M. Hjerkinn, and H. Arnesen, "Long-term influence of diet and/or omega-3 fatty acids on matrix metalloproteinase- 9 and pregnancy-associated plasma protein-A in men at high risk of coronary heart disease," Scandinavian Journal of Clinical and Laboratory Investigation, vol. 68, no. 3, pp. 177-184, 2008.

[65] K. Li, T. Huang, J. Zheng, K. Wu, and D. Li, "Effect of marinederived n-3 polyunsaturated fatty acids on C-reactive protein, interleukin 6 and tumor necrosis factor $\alpha$ : a meta-analysis," PLoS ONE, vol. 9, no. 2, Article ID e88103, 2014.

[66] M. C. Paulo, A. M. Andrade, M. L. Andrade et al., "Influence of n-3 polyunsaturated fatty acids on soluble cellular adhesion molecules as biomarkers of cardiovascular risk in young healthy subjects," Nutrition, Metabolism and Cardiovascular Diseases, vol. 18, no. 10, pp. 664-670, 2008.

[67] O. Eschen, J. H. Christensen, M. T. La Rovere, P. Romano, P. Sala, and E. B. Schmidt, "Effects of marine n-3 fatty acids on circulating levels of soluble adhesion molecules in patients with chronic heart failure," Cellular and Molecular Biology, vol. 56, no. 1, pp. 45-51, 2010.

[68] P. Berstad, I. Seljeflot, M. B. Veierød, E. M. Hjerkinn, H. Arnesen, and J. I. Pedersen, "Supplementation with fish oil affects the association between very long-chain n-3 polyunsaturated fatty acids in serum non-esterified fatty acids and soluble vascular cell adhesion molecule-I," Clinical Science, vol. 105, no. 1, pp. 1320, 2003.

[69] D. Esser, S. J. van Dijk, E. Oosterink, M. Müller, and L. A. Afman, "A high-fat SFA, MUFA, or n3 PUFA challenge affects the vascular response and initiates an activated state of cellular adherence in lean and obese middle-aged men," Journal of Nutrition, vol. 143, no. 6, pp. 843-851, 2013.

[70] Y. Adkins and D. S. Kelley, "Mechanisms underlying the cardioprotective effects of omega-3 polyunsaturated fatty acids," Journal of Nutritional Biochemistry, vol. 21, no. 9, pp. 781-792, 2010.

[71] V. L. Andersen, J. Vogt, T. Obel, J. H. Christensen, and E. B. Schmidt, "The effect of n-3 fatty acids on plasma myeloperoxidase levels in healthy adults," Cellular and Molecular Biology, vol. 56, no. 1, pp. 3-9, 2010.

[72] M. S. Christensen, K. Therkelsen, J. M. Møller, J. Dyerberg, and E. B. Schmidt, "n-3 Fatty acids do not decrease plasma endothelin levels in healthy individuals," Scandinavian Journal of Clinical and Laboratory Investigation, vol. 57, no. 6, pp. 495499, 1997.

[73] K. Schäfer and S. Konstantinides, "Adipokines and thrombosis," Clinical and Experimental Pharmacology and Physiology, vol. 38, no. 12, pp. 864-871, 2011.

[74] D. Tousoulis, A. Plastiras, G. Siasos et al., "Omega-3 PUFAs improved endothelial function and arterial stiffness with a parallel antiinflammatory effect in adults with metabolic syndrome," Atherosclerosis, vol. 232, no. 1, pp. 10-16, 2014. 


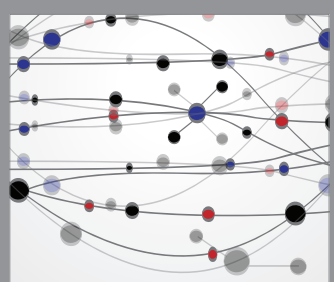

The Scientific World Journal
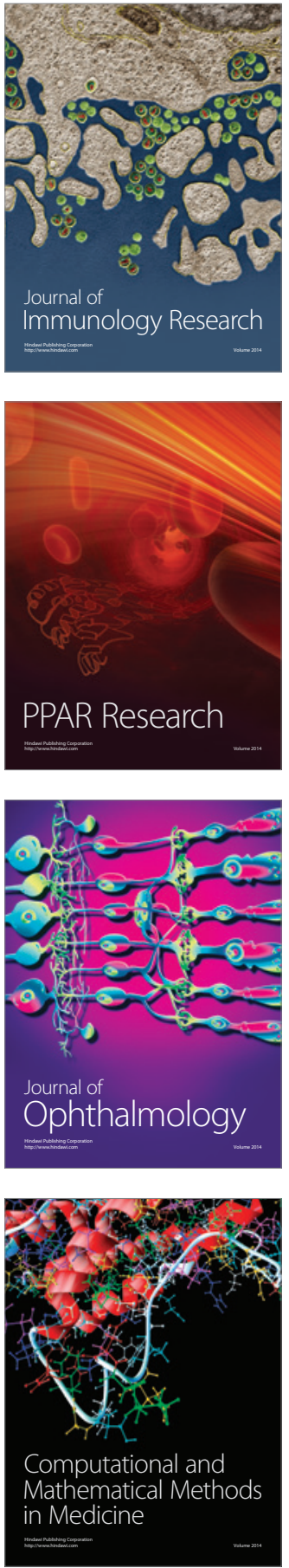

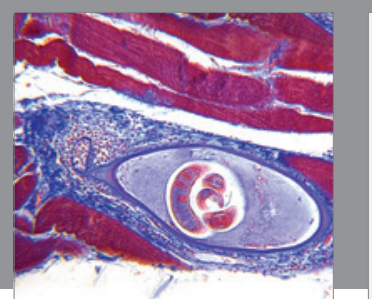

Gastroenterology Research and Practice

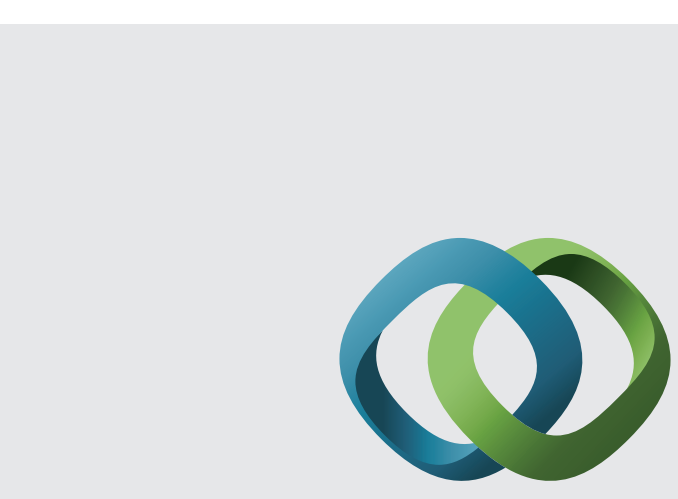

\section{Hindawi}

Submit your manuscripts at

http://www.hindawi.com
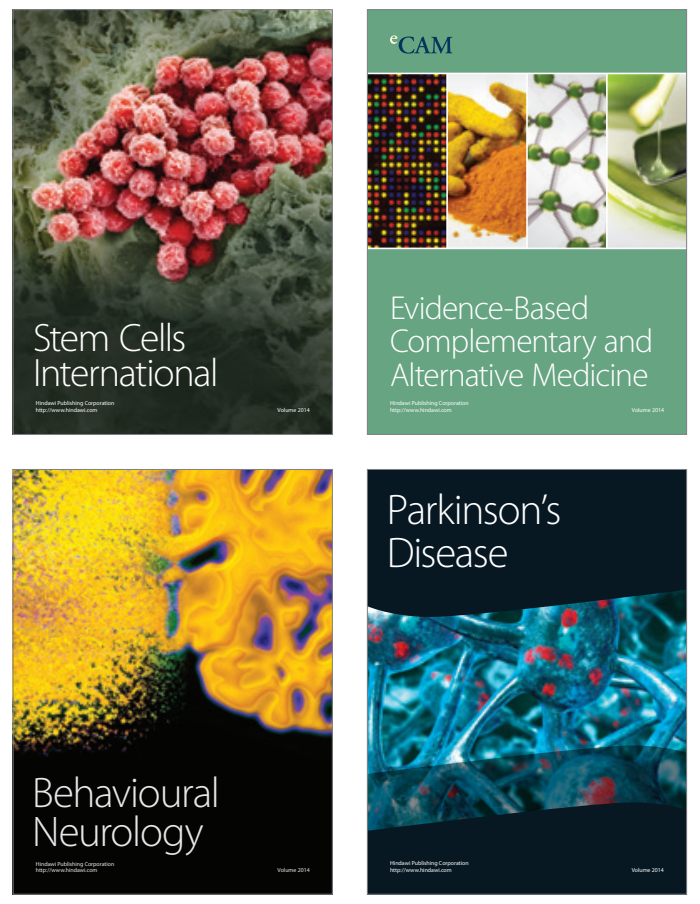
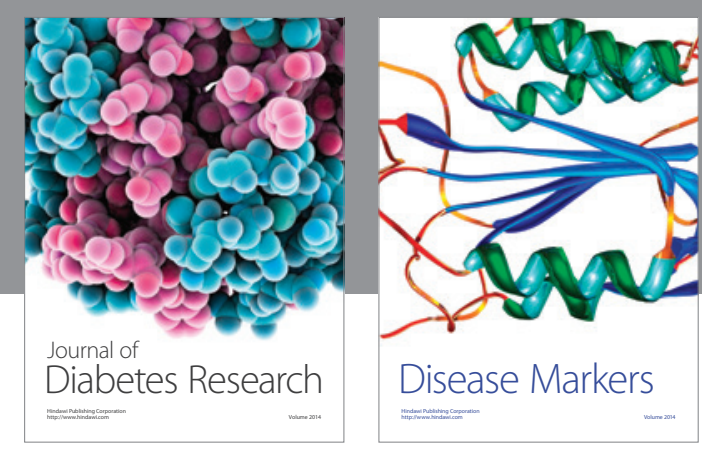

Disease Markers
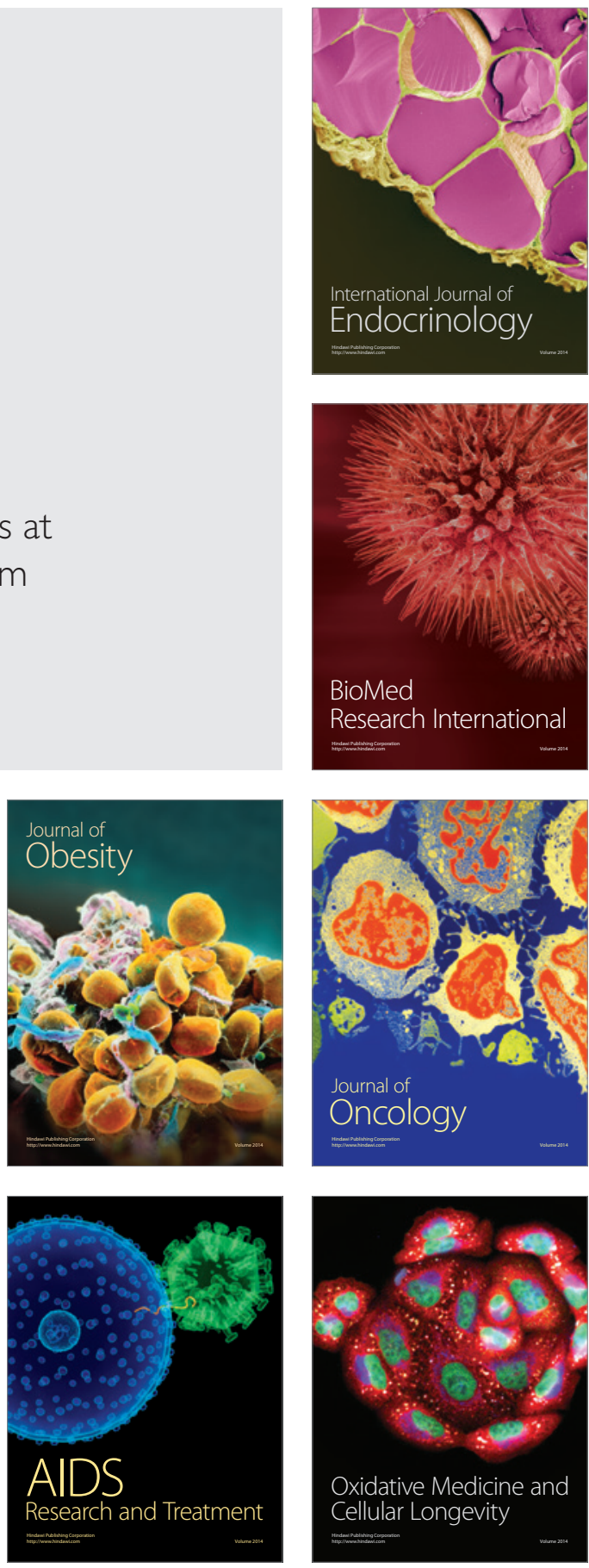